\title{
Impact of COVID-19 Infection on Endocrine Organs
}

\author{
Sattik Siddhanta
}

\begin{abstract}
Coronavirus disease-2019 (COVID-19) has emerged as a pandemic affecting millions of people worldwide. It is characterized by multisystem involvement. The endocrine organs are also expected to be affected in COVID-19 as the interplay between the virus, severe acute respiratory syndrome-coronavirus 2 (SARS-CoV-2), and the endocrine system occurs at multiple organ levels with a swarm of clinical manifestations. In addition to other organ systems, COVID-19 also affects persons with known endocrine disorders, thereby making them susceptible to an increased risk of disease severity, worse prognosis, and increased mortality. However, the virus being a novel one, its possible effect on the endocrine system is not yet entirely understood with limited clinical data to date and remains an emerging area of further research. In this review, the variety of endocrine manifestations that have been reported in both SARS-CoV-1 and SARS-CoV-2 to date have been highlighted. Besides, the possible mechanisms by which SARS-CoV-2 may affect various endocrine organs have been explored.

Keywords: COVID-19, Hormones, Ovary, Pancreas, Pituitary, Testes, Thyroid.

Bengal Physician Journal (2020): 10.5005/jp-journals-10070-7011
\end{abstract}

\section{INTRODUCTION}

Coronavirus disease-2019 (COVID-19) outbreak rapidly emerged as a pandemic with approximately 7.2 million cases and greater than 16 lakh deaths being reported from 220 countries globally till date with the numbers increasing in a geometric progression making it a serious global public health emergency. ${ }^{1}$ The virus has a predilection for multiorgan involvement due to the widespread availability of angiotensin-converting Enzyme 2 receptors, the host receptor for the virus in various organs. ${ }^{2}$ Though COVID-19 infections can be asymptomatic, the primary organ system involved is the respiratory system. Besides the lung, the kidneys, central nervous system, heart, gastrointestinal tract, and coagulation cascade are mostly affected. As a variety of endocrine organs also express ACE2, it is presumed to affect the endocrine system as well though it is relatively rare compared to other systems as evident from the literature search. Although newer manifestations are being appreciated and recognized regularly, the impact of COVID-19 infection on the endocrine system has hardly been reported. Severe acute respiratory syndrome-coronavirus 2 (SARS-CoV-2) has many structural similarities with the original SARS-CoV-1. Hence, it may be presumed that exploring the pathogenesis and the myriad of clinical manifestations may be highly beneficial in understanding the possible effects of the virus on various endocrine organs. ${ }^{3}$ The various adverse effects of the infection on different endocrine organs and their putative mechanisms are discussed in this review article in a nutshell.

\section{Pituitary}

The hypothalamo-pituitary axis (HPA) plays a pivotal role in tackling inflammatory responses during viral infections. The effect on HPA can be a result of either direct or indirect injury. Direct injury may be related to ACE-2 receptor-mediated SARS-CoV-2 infection of neuronal cells, causing cell edema and necrosis. ${ }^{4}$ The indirect effects on HPA are being mediated by various pro-inflammatory cytokines that activate the HPA, thereby resulting in raised serum cortisol levels. ${ }^{5}$ Also, inflammation may result in inflammation of the pituitary, causing hypophysitis in SARS-CoV. However,
Department of General Medicine, IPGMER and SSKM Hospital, Kolkata, West Bengal, India

Corresponding Author: Sattik Siddhanta, Department of General Medicine, IPGMER and SSKM Hospital, Kolkata, West Bengal, India, Phone: +91 9433151126, e-mail: drcalmed@gmail.com

How to cite this article: Siddhanta S. Impact of COVID-19 Infection on Endocrine Organs. Bengal Physician Journal 2020;7(2):35-38.

Source of support: Nil

Conflict of interest: None

in SARS-CoV-1-affected individuals, hypocortisolism has been documented as a frequently encountered delayed complication. Postviral syndromes usually present as fatigue, lassitude, mood disorders, and dizziness; these symptoms improved significantly with physiological doses of cortisosteroid replacement. ${ }^{6}$ Inflammatory cytokine storm syndrome reported in COVID-19 mediated by IL6 is also known to suppress IGF1. Though reduced IGF1 has not been demonstrated, it has been speculated as a possible risk factor for autism in infants of pregnant mothers affected with COVID-19.

Patients with disorders of HPA may often suffer from diabetes insipidus due to posterior pituitary involvement. In these patients, the febrile episodes due to the infection can precipitate insensible water loss, resulting in life-threatening hypernatremia. ${ }^{7}$ Hence, physicians need to be extremely vigilant in this context and counsel the patients accordingly.

\section{THYROID}

A publication on 175 patients affected with SARS-CoV-1 showed reduced serum T3 and FT4 levels. Serum thyrotropin levels were reduced too, thereby suggesting the probabilities of either sick euthyroid syndrome or central hypothyroidism. ${ }^{8}$ In another similar study on the involvement of the thyroid gland during SARS-CoV-1,

\footnotetext{
(c) The Author(s). 2020 Open Access This article is distributed under the terms of the Creative Commons Attribution 4.0 International License (https:// creativecommons.org/licenses/by-nc/4.0/), which permits unrestricted use, distribution, and non-commercial reproduction in any medium, provided you give appropriate credit to the original author(s) and the source, provide a link to the Creative Commons license, and indicate if changes were made. The Creative Commons Public Domain Dedication waiver (http://creativecommons.org/publicdomain/zero/1.0/) applies to the data made available in this article, unless otherwise stated.
} 
three patients had central hypothyroidism, two had subclinical thyrotoxicosis, and a patient had primary hypothyroidism with antithyroid peroxidase (TPO) antibodies. ${ }^{6}$ Sick euthyroid syndrome, also known as "nonthyroidal illness syndrome" (NTI), can occur, which may further aggravate the disease and thereby complicate management strategies. The possible mechanism is that, during any acute illness, there occur significant changes in the binding of thyroid hormones, their uptake at the cellular level, and decreased functioning of the enzyme type I deiodinase, thereby resulting in decreased T4 to T3 conversion. ${ }^{9}$ There also occurs increased degradation of T3 in peripheral tissues due to increased functioning of enzyme type 3 deiodinase. The ultimate net effect is low thyroid hormones (T4 and T3). Downregulation of the hypothalamo-pituitary system was also responsible for the low circulating TSH and FT4 levels.

A histopathological study on the impact of the virus on the thyroid gland revealed severe and widespread injury of the follicular epithelium and parafollicular cells. ${ }^{9}$ These changes resulted in decreased levels of thyroid hormones. Parafollicular cells were absent in tissue specimens from affected patients. Damage to these cells may result in decreased calcitonin production. Wang et al. observed no significant changes in the morphology of the thyroid follicles but noticed some degree of interstitial lymphocytic infiltration. The alterations in the levels of thyroid hormones may persist for many weeks even after clinical recovery. These studies highlight the importance of periodic monitoring of thyroid function tests not only during the acute phase but also during convalescent phases, with the necessity of Levothyroxine therapy if the situation demands. There have been evidence of spontaneous restoration of thyroid function over a span few months in SARS-CoV patients, implying the pertinence of assessment of thyroid function tests of the cured individuals at regular intervals. ${ }^{6}$

\section{Parathyroids}

Several studies have shown a very important role of parathyroid glands during viral fusion of many viruses. In addition, studies have also shown that calcium ions promoted viral replication by directly interacting with the fusion peptides of these enveloped viruses. ${ }^{11}$ Hypocalcemia, though in a mild form, has been already found to be an extremely common finding in patients with SARS. Another retrospective single center study demonstrated a significantly high prevalence of hypocalcemia (80\%) on initial evaluation. The patients were predominantly elderly males with a positive linear correlation between serum calcium, LDH, and PCR levels. In multivariate analyses, hypocalcemia was found to be an independent risk factor for hospitalization; however, it predicted ICU admission, duration of stay, and mortality in univariate analysis. ${ }^{12}$ Since hypocalcemia may have a life-threatening adverse impact on the cardiovascular system, periodic calcium monitoring and adequate supplementation in the proper dosage, if required, is recommended in all admitted patients.

\section{Endocrine Pancreas}

Multiple pathogens, including viruses, have been implicated to cause type I diabetes mellitus in human beings. Coronavirus is one such culprit virus known to cause diabetes mellitus. ${ }^{13}$ The virus enters multiple organs by interacting with the ACE2 receptor. The expression of ACE2 is much stronger and avid in the endocrine pancreas (particularly B and D cells of the islets of Langerhans) in comparison to the exocrine pancreas. ${ }^{14}$ The extent and impact of tissue damage are directly proportional to the magnitude of the level of ACE-2 expression. Hence, the SARS-CoV virus may damage the islet of Langerhans of the pancreas and cause acute onset diabetes mellitus. On the contrary, the incidence of acute pancreatitis was found to be relatively rare. The biopsy findings in patients of SARS-CoV-2 were studied by Yao et al. They reported no obvious abnormalities in the exocrine pancreas but noticed massive degeneration of a few islet cells. ${ }^{15} \mathrm{New}$-onset diabetes occurred in more than half of the cases who had no known history of diabetes mellitus and received no treatment with systemic corticosteroids during the entire course of illness. Hyperglycemia was found to be an important predictor for mortality in patients, both with and without any known history of diabetes mellitus. There was an incremental rise in mortality and morbidity, with increasing FPG. Another very interesting association was between hypoxia and FPG levels. Among patients with no known history of diabetes and before the commencement of steroid therapy, those who had hypoxia $\left(\mathrm{SpO}_{2}<93 \%\right)$ had higher FPG levels compared to those who did not have hypoxia in both the survivor and deceased groups. In both groups, elevated FPG levels started to normalize in the third week irrespective of corticosteroid dosage, thereby indicating restoration of the pancreatic islet cell function. Persons living with uncontrolled diabetes mellitus may present initially with relatively milder symptoms, but they were found to be at an increased risk of rapid progression, catastrophic course of events, with uncontrolled cytokine storm contributing to a worse outcome and guarded prognosis. Wang et al. also proposed that in diabetes mellitus, the acute infection might trigger stress and increase secretion of counterregulatory hormones, such as glucocorticoids, glucagon, and catecholamines, all of which cause elevated fasting and postprandial plasma glucose levels, increased glycemic variability, and higher incidence of microvascular and macrovascular complications, thereby resulting in higher mortality in these group of cases. ${ }^{16}$ Another study demonstrated that greater than $52 \%$ had elevated blood glucose levels with a mean of $7.4 \mathrm{mmol} / \mathrm{L} .{ }^{17}$ Studies have also demonstrated that SARS-CoV-2 patients with diabetes mellitus are at a higher risk of developing ARDS and septic shock with a catastrophic course due to rapid progression.

Clinical therapeutic strategies in COVID-19 patients must consider these factors, and close monitoring of blood glucose levels is necessary for all patients, particularly in diabetic subjects. Given the frequency of dysglycemia in COVID-19, it may be prudent to employ blood glucose screening of all admitted COVID-19 patients to check for new-onset dysglycemia and plan therapeutic strategies accordingly.

\section{Adrenals}

One of the most common culprits of acute adrenal insufficiency is viral pathogens. Stimulation of the Hypothalamo pituitary adrenal (HPA) by proinflammatory cytokines has several effects. It can result in increased perfusion of adrenal glands, thereby increasing the risk of spontaneous hemorrhage. ${ }^{18}$ Secondly, increased steroidogenesis results in immunomodulation towards increased Th2 helper T cell response. Though it is highly beneficial in the acute phase, this dysregulated response often results in a delay in the viral clearing, especially in the protracted phase. ${ }^{18}$ SARS-CoV produces viral peptides that are structurally akin to Adrenocorticotropic hormone (ACTH). The host immune response produces a significant titer of antibodies against these viral proteins that also collaterally destroy host Adrenocorticotropic hormone (ACTH), resulting in acute adrenal insufficiency. ${ }^{19} \mathrm{~A}$ similar mechanism of molecular 
mimicry has been proposed in SARS-CoV-2 infection too. There are adequate expressions of ACE2 receptors on the adrenal glands, thereby reiterating the propensity of the SARS-CoV-2 virus to cause adrenal insufficiency. ${ }^{20}$ Early systematic reviews stressed on the fact that the use of systemic glucocorticoids did not reduce mortality in COVID-19 but resulted in increased hospitalization and delayed viral clearing. Based on these, the World Health Organization had initially recommended against the use of corticosteroids except in refractory shock. ${ }^{21}$ However, recent inferences particularly from the recovery trial have highlighted dramatically significant benefits with steroid use in proper dosage to critically ill COVID-19 patients. The trial clearly demonstrated that dexamethasone reduced the risk of mortality by $17 \%$, particularly in ventilated patients. ${ }^{22}$ Other similar clinical trials are also in the pipeline. Endocrine implications of the above observations and inferences include increased risk for long-term adrenal suppression, susceptibility to infections, and dysglycemia due to an exogenous use of corticosteroids.

A recent study by Tan et al. has shown that severe COVID-19 patients mount a significant degree of adrenal response and that baseline serum cortisol levels were a vital predictor of prognosis in these patients. They suggested a cutoff of $27 \mu \mathrm{g} / \mathrm{dL}(744 \mathrm{nmol} / \mathrm{L})$ as a predictor of 15 -day mortality. ${ }^{23}$ It remains an enigma whether the timing of starting glucocorticoid therapy is critical to extract the beneficial effects of therapy and what other miscellaneous mechanisms may explain this beneficial effect of steroids.

The histolopathological changes in adrenals of deceased patients were described from postmortem biopsy samples by Ding et al. ${ }^{24}$. They found infiltration of the adrenal medulla predominantly by monocytes and lymphocytes. They also noted focal necrosis and not global necrosis of the gland. No such studies have been conducted in SARS-CoV-2 patients to date, and it remains a merging area of active research. However, it may be hypothesized that similar changes in the adrenal gland could happen due to the similar structure of both the viruses and their identical mechanism of entry in the human body.

\section{Gonads}

ACE2 is expressed in significant amounts in both the spermatogonia, Sertoli cells and Leydig cells of the testis. Studies have demonstrated that transmembrane protease serine 2 (TMPRSS2) necessary for viral $S$ protein priming is concentrated by a significant extent in the spermatogonia and spermatids. ${ }^{25}$ These observations suggest the possibility of the testes remaining a high-risk organ vulnerable to damage by SARS-CoV-2 infection. However, unlike testes, similar studies have not been conducted to date in the female counterpart, i.e. ovaries. The presence of orchitis has been reported in SARS infection, and autopsy findings revealed germ-cell destruction with lymphocytic infiltrates in testes. Seminal fluid analysis of males with COVID-19 has shown the presence of SARS-CoV-2, but its clinical implication or relation to infertility is still unknown. Studies have shown low serum testosterone levels in males with COVID-19 infection compared to controls. ${ }^{26}$ Low testosterone levels may serve as an important marker of COVID-19 disease and severity, particularly in elderly male subjects. In these patients, serum luteinizing hormone (LH) levels were elevated, suggesting primary hypogonadism. ${ }^{27}$ Estrogen and its most active form-17 B estradiol-act on various cellular subsets of the immune system by characteristic epigenetic mechanisms, resulting in modulation of lymphocyte number and activity. This may account for relatively less ovarian involvement in females compared to testes in males.

\section{Conclusion}

Although the effects of COVID-19 on multiple organs and organ systems have been studied and reported in the literature, the effects of the virus on the endocrine organs have hardly been explored. The above brief review is mainly theoretical and contains speculations from evidence gathered from studies from SARS-CoV-1 infection and animal studies. A collaborative and multidisciplinary approach will definitely prevent adverse clinical outcomes and improve upon the prognosis in people affected by COVID-19 infection with different endocrine diseases and improve their well-being. Research on the effects of COVID-19 infection on the endocrine system should be of utmost importance and priority, considering the capability for a myriad of effects on various organs of the endocrine system and their diseases by the novel life-threatening virus. It is prudent to mention that a multidisciplinary approach including endocrinologists is the need of the hour for reducing the morbidity and mortality from the disease. Further research is definitely warranted, including the collection of clinical data from widespread sources, histological and postmortem autopsy studies, and basic science studies as well to understand the possible effects of the virus on the very vital endocrine system.

\section{References}

1. Worldometer. Coronavirus cases. https://www.worldometers.info/ coronavirus/. DOI: 10.1101/2020.01.23.20018549V2.

2. Corman VM, Muth D, Niemeyer D, et al. Hosts and sources of endemic human coronaviruses. Adv Virus Res 2018;100:163-188. DOI: 10.1016/ bs.aivir.2018.01.001.

3. Chen N, Zhou M, Dong X, et al. Epidemiological and clinical characteristics of 99 cases of 2019 novel coronavirus pneumonia in Wuhan, China: a descriptive study. Lancet 2020;395(10223):507-513. DOI: 10.1016/S0140-6736(20)30211-7.

4. Wu Y, Xu X, Chen Z, et al. Nervous system involvement after infection with COVID-19 and other coronaviruses. Brain Behav Immun 2020;87:18-22. DOI: 10.1016/j.bbi.2020.03.031.

5. Turnbull AV, Rivier C. Regulation of the HPA axis by cytokines. Brain Behav Immun 1995;9:253-275. DOI: 10.1006/brbi.1995.1026.

6. Leow MK, Kwek DS, Ng AW, et al. Hypocortisolism in survivors of severe acute respiratory syndrome (SARS). Clin Endocrinol (Oxf) 2005;63(2):197-202. DOI: 10.1111/j.1365-2265.2005.02325.x.

7. Kaiser UB, Mirmira RG, Stewart PM (2020) Our response to COVID-19 as endocrinologists and diabetologists. J Clin Endocrinol Metab 2020;105(5):dgaa148. DOI: 10.1210/clinem/dgaa148.

8. Wang W, Ye Y, Yao H, et al. Evaluation and observation of serum thyroid hormone and parathyroid hormone in patients with severe acute respiratory syndrome. J Chin Antituberculous Assoc. 2003;25:232-234.

9. Jonklaas J, Bianco AC, Bauer AJ, et al. Guidelines for the treatment of hypothyroidism: prepared by the American Thyroid Association task force on thyroid hormone replacement. Thyroid 2014;24(12):1670-1751. DOI: 10.1089/thy.2014.0028.

10. Wei $\mathrm{L}$, Sun $\mathrm{S}$, Xu CH, et al. Pathology of the thyroid in severe acute respiratory syndrome. Hum Pathol 2007;38(1):95-102. DOI: 10.1016/j. humpath.2006.06.011.

11. Millet JK, Whittaker GR. Physiological and molecular triggers for SARS-CoV membrane fusion and entry into host cells. Virology 2018;517:3-8. DOI: 10.1016/j.virol.2017.12.015.

12. Di Filippo L, Formenti AM, Rovere-Querini P, et al. Hypocalcemia is highly prevalent and predicts hospitalization in patients with COVID-19. Endocrine 2020;68(3):475-478. DOI: 10.1007/s12020-02002383-5.

13. Navas de Solis C, Foreman JH. Transient diabetes mellitus in a neonatal thoroughbred foal. J Vet Emerg Crit Care 2010;20:611-615. DOI: 10.1111/j.1476-4431.2010.00588.x. 
14. Yang JK, Lin SS, Ji XJ, et al. Binding of SARS coronavirus to its receptor damages islets and causes acute diabetes. Acta Diabetol 2010;47:193-199. DOI: 10.1007/s00592-009-0109-4.

15. Yao XH, Ty $L, Z c H$, et al. Histopathological study of new coronavirus pneumonia (COVID-19) in three patients. Chin J Pathol 2020;49(5):411-417. DOI: 10.3760/cma.j.cn112151-2020031200193.

16. Wang A, Zhao W, Xu Z, et al. Timely blood glucose management for the outbreak of 2019 novel coronavirus disease (COVID-19) is urgently needed. Diabetes Res Clin Pract 2020;162:108118. DOI: 10.1016/j. diabres.2020.108118.

17. Chen N, Zhou M, Dong $X$, et al. Epidemiological and clinical characteristics of 99 cases of 2019 novel coronavirus pneumonia in Wuhan, China: a descriptive study. Lancet 2020;395:507-513. DOI: 10.1016/S0140-6736(20)30211-7.

18. Paolo WF Jr, Nosanchuk JD. Adrenal infections. Int J Infect Dis 2006;10:343-353. DOI: 10.1016/j.ijid.2005.08.001.

19. Wheatland R. Molecular mimicry of ACTH in SARS - implications for corticosteroid treatment and prophylaxis. Med Hypotheses 2004;63:855-862. DOI: 10.1016/j.mehy.2004.04.009.

20. Turner AJ, Hooper NM. 84-Angiotensin-converting enzyme 2. In: Barrett AJ, Rawlings ND, Woessner JF, eds. Handbook of Proteolytic Enzymes, 2nd ed. London: Academic Press; 2004. pp. 349-512.
21. World Health Organisation. Clinical care of severe acute respiratory infections - Tool kit. Available from: https://www.who.int/ publicationsdetail/clinical care of severe acute respiratory infections tool kit [Last accessed on May 25, 2020].

22. Beigel JH, Tomashek KM, Dodd LE, et al. Remdesivir for the Treatment of Covid-19_Final Report. N Engl J Med. 2020;383(19):1813-1826. DOI: 10.1056/NEJMoa2007764

23. Tan T, Khoo B, Mills EG, et al. Association between high serum total cortisol concentrations and mortality from COVID-19. Lancet Diabetes Endocrinol 2020;8:659-660. DOI: 10.1016/S2213-8587(20)30216-3.

24. Ding $Y$, Wang $H$, Shen $H$, et al. The clinical pathology of severe acute respiratory syndrome (SARS): a report from China. J Pathol 2003;200:282-289. DOI: 10.1002/path.1440.

25. Wang Z, Xu X. scRNA-seq profiling of human testes reveals the presence of the ACE2 receptor, a target for SARS-CoV-2 infection in spermatogonia, Leydig and Sertoli cells. Cells 2020;9(4):920. DOI: 10.3390/cells9040920.

26. Li D, Jin $M, B a o P$, et al. Clinical characteristics and results of semen tests among men with coronavirus disease 2019. JAMA Netw Open 2020;3:e208292. DOI: 10.1001/jamanetworkopen.2020.8292.

27. Ma L, Xie W, Li D, et al. Effect of SARS CoV-2 infection upon male gonadal function: a single center based study. medRxiv 2020. DOI: $10.1101 / 2020.03 .21 .20037267$. 